\title{
Enhancement in hole current density on polarization in poly(3- hexylthiophene):cadmium selenide quantum dot nanocomposite thin films
}

\author{
Kusum Kumari, ${ }^{1, a)}$ Suresh Chand, ${ }^{2}$ V. D. Vankar, ${ }^{1}$ and Vikram Kumar ${ }^{2}$ \\ ${ }^{1}$ Department of Physics, Nanoscience and Nanotechnology Laboratory, Indian Institute of Technology Delhi, \\ New Delhi 110 016, India \\ ${ }^{2}$ Material Division, National Physical Laboratory, Dr. K. S. Krishnan Road, New Delhi 110 012, India
}

(Received 9 February 2009; accepted 2 May 2009; published online 26 May 2009)

\begin{abstract}
We demonstrate the effect of polarization on space charge limited $J-V$ behavior in poly(3-hexylthiophene) (P3HT):cadmium selenide (CdSe) $(\sim 5 \mathrm{~nm})$ quantum dot nanocomposite thin films in hole-only device configuration, indium tin oxide/poly(ethylenedioxthiophene):polystyrenesulphonate/P3HT:CdSe/Au. Current density has been found to enhance in these hybrid films on polarization. This has been attributed to decrease in characteristic trap energy from 32 to $27 \mathrm{meV}$, trap density from $1.7 \times 10^{18}$ to $1 \times 10^{18} \mathrm{~cm}^{-3}$ and increase in hole mobility from $2.6 \times 10^{-6}$ to $7.7 \times 10^{-6} \mathrm{~cm}^{2} \mathrm{~V}^{-1} \mathrm{~s}^{-1}$ due to field induced enhanced order by dipolar alignment and/or trapping of charge carriers at the nanoscale interfacial boundaries of P3HT and CdSe quantum dots. (C) 2009 American Institute of Physics. [DOI: 10.1063/1.3142393]
\end{abstract}

Conjugated polymers, such as poly(3-hexylthiophene) (P3HT), poly(3-octylthiophene), poly(2-methoxy-5(2ethylhexyloxy)-1,4-phenylinevinylene, etc., dispersed with various inorganic nanoparticles/quantum dots, such as cadmium selenide (CdSe), cadmium telluride, cadmium selenide telluride, zinc oxide, titanium dioxide, etc., are finding applications $^{1-5}$ in the development of organic solar cells. Among various organic-inorganic composites, the most promising candidate is $\mathrm{CdSe}$ nanoparticles dispersed in P3HT polymer. Power conversion efficiency up to $\sim 2.6 \%$ have already been realized ${ }^{6-8}$ in P3HT:CdSe nanocomposites. However, to improve further the efficiency of these devices, it is of utmost importance to understand and reveal fundamental facets related to their charge transport and device physics properties. In fact, innovative ideas are required to modulate/improve the charge transport and device parameters of these hybrid combinations to achieve better performance. Keeping this objective in view, we have used a concept of polarizing the P3HT:CdSe nanocomposite thin films prior to studying their current-voltage $(J-V)$ characteristics. In this polarization process, a fixed field $\left(\sim 3 \times 10^{5} \mathrm{~V} / \mathrm{cm}\right)$ was applied across the composite sample at an elevated temperature $(290 \mathrm{~K})$ and subsequently cooled down to lower temperature (up to $150 \mathrm{~K}$ ) in the presence of the applied field. The field was then removed and the sample was short circuited for a few minutes to remove the stray charges present, if any. After this, the $J-V$ measurements were carried out in hybrid samples at different temperatures by upwardly raising the temperature in the range $150-290 \mathrm{~K}$. To be more precise, $J-V$ characteristics of $\mathrm{P} 3 \mathrm{HT}$ :CdSe quantum dot composite thin films $(\sim 180 \mathrm{~nm})$ were studied in hole-only device configuration, i.e., indium tin oxide (ITO)/ poly(ethylene-dioxythiophene):polystyrenesulphonate (PEDOT:PSS)/P3HT:CdSe/Au, without and with polarization. Interestingly, it has been found that there is a considerable enhancement in current density in the composite films on polarization. These important results on hole conduction

\footnotetext{
${ }^{\text {a) }}$ Author to whom correspondence should be addressed. Electronic mail: kusumiitd@gmail.com.
}

and modulation of charge transport parameters by polarization in P3HT:CdSe nanocomposite films are discussed in this letter.

Two samples, one unpolarized (designated as device 1) and other polarized (designated as device 2), of P3HT:CdSe composite films were prepared under identical conditions in hole-only device configurations, i.e., ITO $(\phi \sim 4.8 \mathrm{eV}) / \mathrm{PEDOT}: \operatorname{PSS}(80 \mathrm{~nm}) / \mathrm{P} 3 \mathrm{HT}: \mathrm{CdSe}$ $(\sim 180 \mathrm{~nm}) / \mathrm{Au}(\phi \sim 5.2 \mathrm{eV})$, as shown in Fig. 1(a). A thin film of PEDOT:PSS (Aldrich USA) was spin coated onto precleaned and plasma treated ITO (sheet resistance $\sim 18 \Omega /$ sq.) coated glass substrates at $2000 \mathrm{rpm}$ and cured at $120{ }^{\circ} \mathrm{C}$ for $30 \mathrm{~min}$ in vacuum. P3HT polymer (Aldrich USA) was used as such without any further purification. CdSe quantum dots $(\sim 5 \mathrm{~nm})$ were synthesized by well known wet chemical route ${ }^{9}$ using $\mathrm{CdO}$, Se powder as precur-

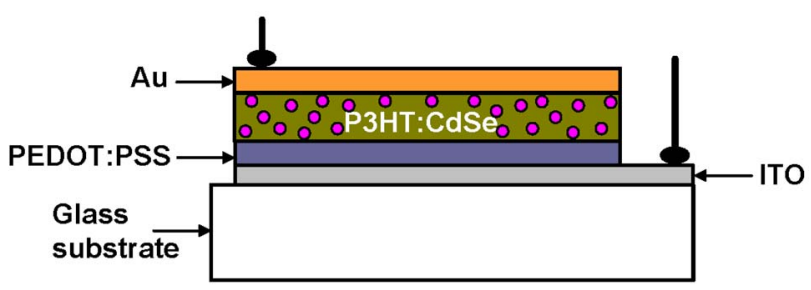

(a)

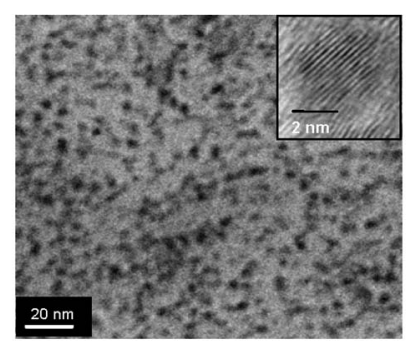

(b)

FIG. 1. (Color online) (a) Device configuration, ITO/PEDOT:PSS/ P3HT:CdSe/Au, and (b) TEM image of CdSe quantum dots dispersed in $\mathrm{P} 3 \mathrm{HT}$ matrix in 1:1 weight ratio. 


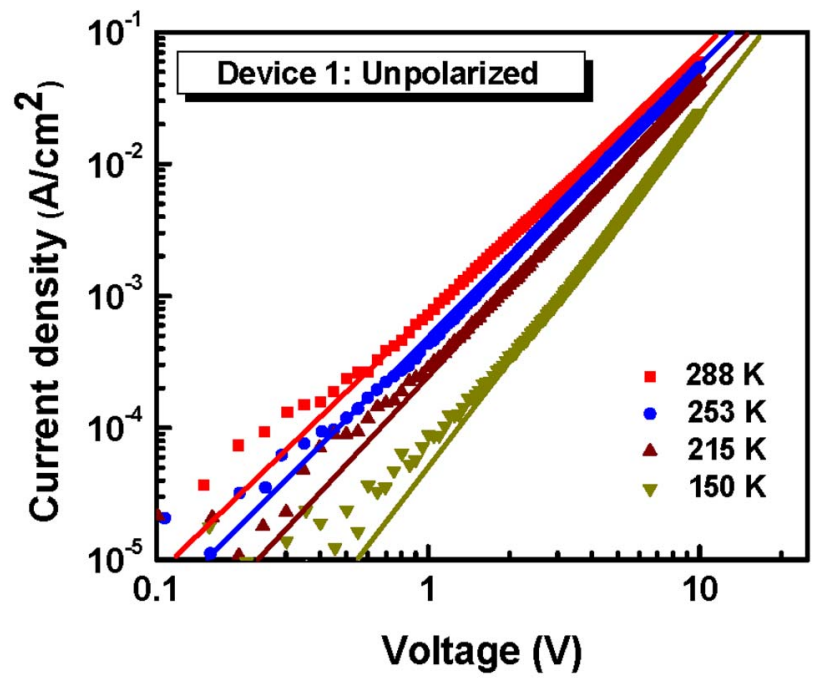

FIG. 2. (Color online) $J-V$ characteristics of device 1 (unpolarized) in the temperature range 150-288 K. Experimental curves (symbols) and theoretical curves (solid lines) calculated using Eq. (5). The values of fitting parameters being $N_{v}=1 \times 10^{19} \mathrm{~cm}^{-3}, \quad H_{b}=1.7 \times 10^{18} \mathrm{~cm}^{-3}, \quad \mu=2.5$ $\times 10^{-6} \mathrm{~cm}^{2} \mathrm{~V}^{-1} \mathrm{~s}^{-1}, T_{c}=370 \mathrm{~K}$, and $E_{t}=32 \mathrm{meV}$.

sor, and trioctyl phosphene oxide (TOPO) as capping agent. To achieve better charge transport properties between the CdSe quantum dots and the host polymer P3HT, capping agent TOPO was removed by treatment with pyridine. For this, the passivated CdSe particles were washed several times in methanol, dissolved in pyridine, and precipitated with hexane. ${ }^{1,7}$ Subsequently, a homogeneous solution was prepared by dissolving $\mathrm{P} 3 \mathrm{HT}$ in chloroform with material content of $10 \mathrm{mg} / \mathrm{ml}$ and dispersing CdSe quantum dots $(1: 1$ weight ratio) in binary solvent of chloroform: pyridine (9:1 volume ratio). Figure 1(b) shows the transmission electron microscopy (TEM) image of CdSe quantum dots dispersed in host P3HT in 1:1 weight ratio. TEM image reveals monodispersion and uniform distribution of CdSe quantum dots in P3HT matrix with an average particle size $\sim 5 \mathrm{~nm}$. Further the inset in top right shows the high resolution TEM of the CdSe quantum dots dispersed in the polymer composite, which shows the presence of corresponding lattice planes and their crystalline nature. Such P3HT:CdSe composite thin films were spin cast in an inert atmosphere onto the PEDOT:PSS films at $2000 \mathrm{rpm}$, and cured at $120^{\circ} \mathrm{C}$ for $15 \mathrm{~min}$. On top of these films, Au electrodes $\sim 400 \mathrm{~nm}$ were deposited by vacuum thermal evaporation at $5 \times 10^{-6}$ Torr. The holeonly devices so obtained were sealed using UV irradiated epoxy resin in inert atmosphere. The $J-V$ characteristics of these samples in the temperature range of 150-290 K were measured upwardly, i.e., from lower to higher temperatures, using a low temperature setup coupled with Keithley 2400 source meter unit interfaced with a computer.

Figure 2 shows the $J$ - $V$ characteristics of device 1 at different temperatures in the range 150-288 $\mathrm{K}$, which has been analyzed in terms of space charge limited current (SCLC) using the trap model ${ }^{10-13}$ considering the exponentially distribution of traps in energy and space described as

$$
n(E)=\left(H_{b} / E_{t}\right) \exp \left(-E / E_{t}\right),
$$

or expressed in another form as

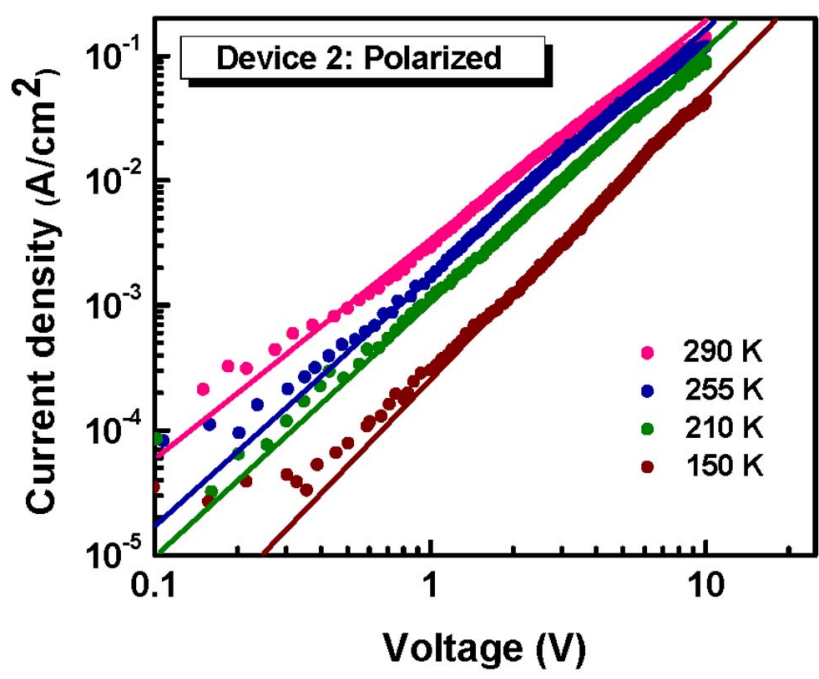

FIG. 3. (Color online) $J-V$ characteristics of device 2 (polarized) in the temperature range 150-290 K. Experimental curves (symbols) and theoretical curves (solid lines) calculated using Eq. (5). The values of fitting parameters being $N_{v}=1 \times 10^{19} \mathrm{~cm}^{-3}, \quad H_{b}=1 \times 10^{18} \mathrm{~cm}^{-3}, \quad \mu=7.7$ $\times 10^{-6} \mathrm{~cm}^{2} \mathrm{~V}^{-1} \mathrm{~s}^{-1}, T_{c}=310 \mathrm{~K}$, and $E_{t}=27 \mathrm{meV}$.

$$
N(E)=H_{b} \exp \left(-E / E_{t}\right),
$$

where $n(E)$ is the distribution function of hole trap density at an energy level $E$ above the valence band edge (assuming uniform spatial distribution), $N(E)=n(E) E_{t}$ is the hole trap density at an energy level $E$ above the valence band edge, $H_{b}$ is the density of traps at the edge of valence band, and $E_{t}$ is characteristic trap energy. $E_{t}$ is also often expressed in terms of the characteristics temperature of trap distribution $T_{c}\left(E_{t}\right.$ $\left.=k_{B} T_{c}\right)$ and $l=E_{t} / k_{B} T=T_{c} / T$, where $k_{B}$ is the Boltzmann's constant.

For the case when the trap energy $E=E_{t}$, Eq. (2) becomes $N\left(E_{t}\right)=H_{b} / e$, which demonstrates that $E_{t}$ characterizes the exponential trap distribution and defines the energy level where the trap density has been reduced by $1 / e$ of its value at the valence band edge. Hence, the characteristic width of the exponential distribution is set by the value of $E_{t}$.

In case of such an exponential distribution of traps, assuming that the trapped hole carrier density $\left(p_{t}\right) \gg$ free hole carrier density $(p)$ and using continuity equation and boundary condition as

$$
\begin{aligned}
& J=q \mu p(x) F(x), \\
& V=\int F(x) d x,
\end{aligned}
$$

the $J$ - $V$ characteristic is governed by ${ }^{14,15}$

$$
J=q^{1-l} \mu N_{v}\left(\frac{2 l+1}{l+1}\right)^{l+1}\left(\frac{l}{l+1} \frac{\varepsilon \varepsilon_{0}}{H_{b}}\right)^{l} \frac{V^{l+1}}{d^{2 l+1}},
$$

where $J$ is the current density, $V$ is the applied voltage, $q$ is the elementary charge, $d$ is the thickness of the material film, $\mu$ is the hole carrier mobility, $F(x)$ is the electric field, $N_{v}$ is the effective density of states, $\varepsilon$ is the dielectric constant of material, and $\varepsilon_{0}$ is permittivity of the free space.

It is seen from Fig. 2 that the experimental data at all temperatures from 150 to $288 \mathrm{~K}$ show good agreement to the above mentioned SCLC trap model. Symbols represent the experimental data and solid lines represent the theoreti- 
TABLE I. Charge (hole) transport parameters for device 1 (unpolarized) and device 2 (polarized) P3HT:CdSe quantum dot nanocomposite thin films.

\begin{tabular}{lcc}
\hline \hline \multicolumn{1}{c}{ Transport parameters } & Device 1 & Device 2 \\
\hline$N_{v}\left(\mathrm{~cm}^{-3}\right)$ & $1 \times 10^{19}$ & $1 \times 10^{19}$ \\
$H_{b}\left(\mathrm{~cm}^{-3}\right)$ & $1.7 \times 10^{18}$ & $1 \times 10^{18}$ \\
$T_{c}(\mathrm{~K})$ & 370 & 310 \\
$E_{t}(\mathrm{meV})$ & 32 & 27 \\
$\mu\left(\mathrm{cm}^{2} \mathrm{~V}^{-1} \mathrm{~s}^{-1}\right)$ & $2.6 \times 10^{-6}$ & $7.7 \times 10^{-6}$ \\
\hline \hline
\end{tabular}

cally generated curves using Eq. (5) at different temperatures. The values of fitting parameters obtained for this case being $N_{v}=1 \times 10^{19} \mathrm{~cm}^{-3}, H_{b}=1.7 \times 10^{18} \mathrm{~cm}^{-3}, \mu=2.5$ $\times 10^{-6} \mathrm{~cm}^{2} \mathrm{~V}^{-1} \mathrm{~s}^{-1}, T_{c}=370 \mathrm{~K}$, and $E_{t}=32 \mathrm{meV}$.

Figure 3 shows the $J-V$ characteristics of device 2 at different temperatures in the range 150-290 K. This experimental data has also been analyzed using trap model as mentioned above. Here, it was also found that the data fits well with the trap model for all temperatures. However, in this case various transport parameters being $N_{v}=1 \times 10^{19} \mathrm{~cm}^{-3}$, $H_{b}=1 \times 10^{18} \mathrm{~cm}^{-3}, \mu=7.7 \times 10^{-6} \mathrm{~cm}^{2} \mathrm{~V}^{-1} \mathrm{~s}^{-1}, T_{c}=310 \mathrm{~K}$, and $E_{t}=27 \mathrm{meV}$.

For comparison sake, transport parameters obtained for devices 2 and 1 are tabulated in Table I. It is explicitly seen from this table that in device 2 the respective values of $H_{b}$, $T_{c}$, and $E_{t}$ decrease, whereas that of $\mu$ increase as compared to their corresponding values in device 1 . Such a modulation of transport parameters on polarization in the composite films has been explained in terms of net reduction in the value of $N\left(E_{t}\right)$ (responsible for controlling trap limited SCLC in P3HT:CdSe composite) indicating transport energy levels becoming shallower. This all in turn is responsible for the significant finding of this work, i.e., considerable enhancement in current density in P3HT:CdSe composite thin films on polarization as seen explicitly from the $J$ - $V$ curves of Fig. 2 (device 1) and Fig. 3 (device 2) at temperature $\sim 290 \mathrm{~K}$ that have been replotted in Fig. 4.

Further, the mechanism/physics responsible for positive change in transport parameters and improvement in the current density has been explained below. When the sample is polarized by applying a fixed electric field at an elevated temperature and cooled down to lower temperatures in the

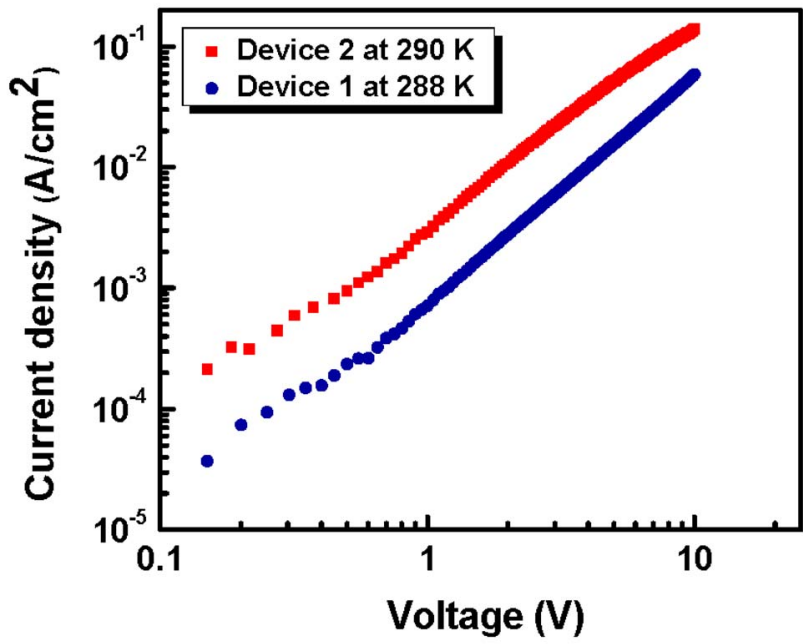

FIG. 4. (Color online) Comparison of $J-V$ characteristics of devices 1 and 2 at temperature $\sim 290 \mathrm{~K}$. presence of the applied field it results in the frozen state in the sample in the form of built-up of a well known internal polarization electric field (electret field). In this case, the mechanism for the origin of this electret field can be understood either (i) in terms of orientation and freezing of permanent dipoles of the individual P3HT and CdSe quantum dot constituents of the composite or dipoles constituted by combination of these components or (ii) the displacement of charge carriers at nanoscale distances and their subsequent trapping at the interface boundaries of the two constituents. In either case, the electret field will not only effect in reducing the trap states in the material but also result in increase in the hole mobility due to enhanced order and in turn enhancement in the current density as found in the present investigations.

In conclusion, it is established that polarization (electret field induced enhanced order) plays a significant role in controlling the hole transport in P3HT:CdSe composite thin films. Though charge transport, in the two cases, is well explained by SCLC trap model with exponential distribution of traps in energy and space yet on polarization the transport parameters $H_{b}, T_{c}$, and $E_{t}$, reduce from their respective values from $1.7 \times 10^{18}$ to $1 \times 10^{18} \mathrm{~cm}^{-3}, 370$ to $310 \mathrm{~K}$, and 32 to $27 \mathrm{meV}$ and $\mu$ increases from $2.5 \times 10^{-6}$ to 7.7 $\times 10^{-6} \mathrm{~cm}^{2} \mathrm{~V}^{-1} \mathrm{~s}^{-1}$. It all in turn results in enhancement in the current density in polarized P3HT:CdSe composite films. These effects have been attributed to the enhanced order due to built up of internal polarization field either due to dipolar effect or due to displacement of charge carriers at nanoscale distances and their subsequent trapping at interface of amorphous-crystalline boundaries of the organic-inorganic constituents. In essence, this letter is an important fundamental contribution on charge transport aspect of P3HT:CdSe composites that reveals the modulation of properties in it which will prove beneficial for the development of organic solar cells based on these hybrid composites as there is considerable enhancement in current density on polarization.

${ }^{1}$ W. U. Huynh, J. J. Dittmer, W. C. Libby, G. L. Whiting, and A. P. Alivisatos, Adv. Funct. Mater. 13, 73 (2003).

${ }^{2}$ W. J. E. Beek, M. M. Wienk, and R. A. J. Janssen, Adv. Mater. (Weinheim, Ger.) 16, 1009 (2004).

${ }^{3}$ T. Shiga, K. Takechi, and T. Motohiro, Sol. Energy Mater. Sol. Cells 90, 1849 (2006).

${ }^{4}$ C. Y. Kwong, W. C. H. Choy, A. B. Djurisic, P. C. Chui, K. W. Cheng, and W. K. Chan, Nanotechnology 15, 1156 (2004).

${ }^{5}$ Y. Zhou, Y. Li, H. Zhong, J. Hou, Y. Ding, C. Yang, and Y. Li, Nanotechnology 17, 4041 (2006).

${ }^{6}$ B. Sun and N. C. Greenham, Phys. Chem. Chem. Phys. 8, 3557 (2006).

${ }^{7}$ W. U. Huynh, J. J. Dittmer, and A. P. Alivisatos, Science 295, 2425 (2002).

${ }^{8}$ N. C. Greenham, X. Peng, and A. P. Alivisatos, Phys. Rev. B 54, 17628 (1996).

${ }^{9}$ L. Qu, W. W. Yu, and X. Peng, Nano Lett. 4, 465 (2004).

${ }^{10}$ A. K. Kapoor, S. C. Jain, J. Pootmans, V. Kumar, and R. Mertens, J. Appl. Phys. 92, 3835 (2002).

${ }^{11}$ N. F. Mott and R. W. Gurney, Electronic Processes in Ionic Crystals (Oxford, London, 1940).

${ }^{12}$ A. R. Brown, D. D. C. Bradley, J. H. Burroughes, R. H. Friend, N. C. Greenham, P. L. Burn, A. B. Holmes, and A. Kraft, Appl. Phys. Lett. 61, 2793 (1992).

${ }^{13}$ Z. Chiguvare and V. Dyakonov, Phys. Rev. B 70, 235207 (2004)

${ }^{14}$ V. Kumar, S. C. Jain, A. K. Kapoor, J. Pootmans, and R. Mertens, J. Appl. Phys. 94, 1283 (2003).

${ }^{15}$ K. C. Kao and W. Hwang, Electrical Transport in Solids (Pergamon, Oxford, 1981), p. 160. 